\title{
Erratum to: Hepatocyte glutathione peroxidase-1 deficiency improves hepatic glucose metabolism and decreases steatohepatitis in mice
}

\author{
Troy L. Merry ${ }^{1,2} \cdot$ Melanie Tran $^{1} \cdot$ Garron T. Dodd $^{1} \cdot$ Salvatore P. Mangiafico $^{3}$. \\ Florian Wiede $^{1} \cdot$ Supreet Kaur $^{1}$ - Catriona L. McLean ${ }^{4} \cdot$ Sofianos Andrikopoulos $^{3}$. \\ Tony Tiganis ${ }^{1}$
}

Published online: 14 October 2016

(C) Springer-Verlag Berlin Heidelberg 2016

\section{Erratum to: Diabetologia \\ DOI 10.1007/s00125-016-4084-3}

Unfortunately, 'pyruvate dehydrogenase kinase 2' was incorrectly written as 'pyruvate kinase 2 ' in the penultimate paragraph of this paper. The correct sentence reads:

'We speculate that the oxidation of such PTPs would promote insulin signalling to regulate hepatic glucose metabolism, although we cannot exclude the contribution of other pathways given the growing number of metabolic proteins that can be oxidised, including pyruvate kinase M2 [49] and pyruvate dehydrogenase kinase 2 [50].'

The online version of the original article can be found at http://dx.doi. org/10.1007/s00125-016-4084-3.

Tony Tiganis

Tony.Tiganis@monash.edu

1 Metabolic Disease and Obesity Program and Department of Biochemistry and Molecular Biology, Biomedicine Discovery Institute, Monash University, Melbourne, VIC 3800, Australia

2 Present address: Faculty of Medical and Health Sciences, The University of Auckland, Aukland, New Zealand

3 Department of Medicine (Austin Hospital), The University of Melbourne, Melbourne, VIC, Australia

4 Department of Anatomical Pathology, Alfred Hospital, Prahran, VIC, Australia 